\title{
A Theoretical Calculation Method of Influence Radius of Settlement Based on the Slices Method in Tunnel Construction
}

\author{
Xiaoxu Tian $\mathbb{D}^{1},{ }^{1,2}$ Zhanping Song $\mathbb{D}^{1,2}$ Bing Wang, ${ }^{1,2}$ and Guannan Zhou ${ }^{3}$ \\ ${ }^{1}$ School of Civil Engineering, Xi'an University of Architecture and Technology, Xi'an, Shaanxi 710055, China \\ ${ }^{2}$ Shaanxi Key Laboratory of Geotechnical and Underground Space Engineering, Xi'an 710055, China \\ ${ }^{3}$ China Railway Construction Bridge Engineering Bureau Group Co., Ltd., Tianjin 300300, China
}

Correspondence should be addressed to Zhanping Song; songzhpyt@xauat.edu.cn

Received 3 August 2019; Revised 25 September 2019; Accepted 10 October 2019; Published 25 January 2020

Academic Editor: José António Fonseca de Oliveira Correia

Copyright (c) 2020 Xiaoxu Tian et al. This is an open access article distributed under the Creative Commons Attribution License, which permits unrestricted use, distribution, and reproduction in any medium, provided the original work is properly cited.

\begin{abstract}
At present, the empirical formula is used to calculate the influence radius of surface settlement and the width of settlement trough, which lacks theoretical support. Aiming at this problem, this paper derived the theoretical calculation formula for predicting the influence radius of formation settlement based on the slices method. Then, the expression of the width of settlement trough was obtained according to the relationship between the settlement influence radius and the settlement trough width. The rationality of the formula was verified by the Heathrow Express tunnel and the Green Park tunnel. Through analysis and discussion, it was found that in the clay stratum, the settlement calculation formula can more accurately predict the surface settlement, while there is a big error in predicting the stratum settlement within $4 d$ near the tunnel vault. In the sand layer, the internal friction angle is less than $40^{\circ}$, and the reinforcement surface is applied to the unsupported face to reduce the radius of influence; in the clay formation, when the cohesion is less than $50 \mathrm{kPa}$, the influence radius can be reduced by applying reinforcement measure to the unsupported face.
\end{abstract}

\section{Introduction}

The underground excavation construction of urban subway tunnel will lead to the deformation of strata settlement and then affect the safety and stability of adjacent existing buildings within the settlement range of strata. If the formation is too large, it may cause damage to the building [1-5]. Therefore, it is of great engineering significance to predict the range of formation settlement and settlement caused by the underground excavation of urban subway tunnels.

At present, there are many methods for predicting surface settlement caused by urban subway tunnel construction, such as the empirical formula method [6-8], elastic strain method [9-13], air stress function method [14-18], stochastic medium theory [19-21], numerical simulation method $[22,23]$, and centrifugal test method $[24,25]$. Although there are many methods for predicting surface settlement, the calculation of the influence radius of formation settlement is still mainly based on empirical formula data regression [26-29], and the settlement trough width is not theoretically determined. However, the empirical formula has a large degree of subjectivity. Engineers with different engineering conditions may get different results. When the regression data obtained from other projects are applied to a certain project, it is still not possible to accurately predict the settlement and influence radius of settlement due to the complexity of soil mass. The key to studying the law of settlement deformation is to determine the width of the settlement trough, which also is the focus of many scholars. Therefore, it is necessary to theoretically study the settlement deformation law of the overlying soil caused by the underground excavation construction of urban subway tunnels, so as to objectively predict the influence range of settlement and settlement.

Based on the aforementioned problems, this paper used the slices method of slope stability analysis to analyze the soil 
of tunnel excavation disturbance range and derived the theoretical calculation formula of the influence radius of settlement. Then, the expression of the width of settlement trough was obtained according to the relationship between the settlement influence radius and the settlement trough width. The rationality of the formula was verified by the Heathrow Express tunnel and the Green Park tunnel. At the same time, the influence radius of the settlement of sand and clay stratum, frequently encountered in the construction of urban subway tunnels, as well as the measures to reduce the radius of settlement and maximum surface settlement are analyzed. The research methods and results of this paper provide a reference for the theoretical calculation of the influence radius of settlement caused by the underground excavation of urban subway tunnels.

\section{Theoretical Calculation of Influence Radius of Settlement}

At present, there are many theoretical studies on the failure modes of the arch crown and the face, for example, Janssen silos theory, rock failure mode, parabolic failure mode [30, 31], and logarithmic spiral mode [32]. However, in actual engineering, the failure mode of the excavation face is far from the analysis model above. In order to find out the true failure mode of the excavation face, Mair [33] used the centrifuge model test to prove that the failure surface of the cohesive soil layer is extended upward and towards two sides, and it is like a basin, narrow in the lower part and wide in the upper part. Selby [34] also shows that the failure surface of the sandy soil layer is narrow chimney shaped that directly develops to the surface of the tunnel from both sides. The actual failure mode of the excavation surface is consistent with the test results of Mair. In fact, for shallow tunnels in sand and clay, the effects of excavation will extend to the surface, failing to form a "natural arch." After the tunnel is excavated, the rock and soil will collapse and move, which affects the surface and forms a collapsed area [35, 36]. The failure pattern is shown in Figure 1.

The excavation of the soil mass on the tunnel face will break the original stress balance state of surrounding soil mass, and the disturbed soil mass will deform towards the tunnel excavation face under the action of gravity. Due to construction methods and other factors, the volume of soil mass actually excavated is often larger than the volume of tunnel designed for excavation. The overexcavated part of the soil is called formation loss [17] (see Figure 1).

\section{Peck Formula and the Width of the Settlement Trough}

After the tunnel lining is closed into a ring, the overlying soil is layered and filled into the overexcavated part, which eventually causes surface settlement. Peck summarized a large number of measured data of surface settlement caused by tunnel construction and believed that surface settlement accords with normal distribution law [4] and proposed the famous Peck formula as follows:

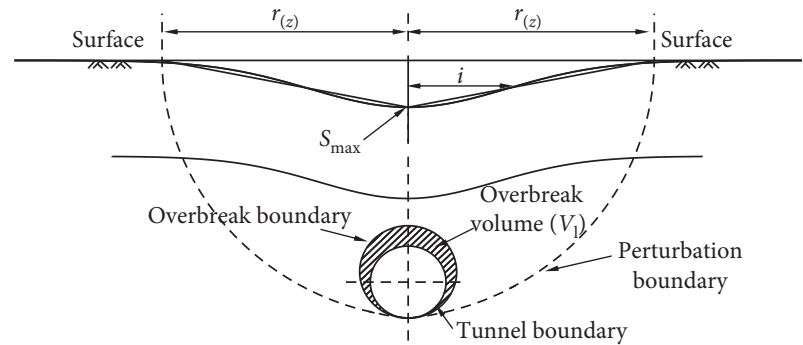

FIgURE 1: Stratum settlement caused by tunnelling.

$$
S_{(x)}=S_{\max } \exp \left(-\frac{x^{2}}{2 i^{2}}\right),
$$

where $S_{(x)}$ is the settlement value of the distance from the surface of the tunnel to the central axis of the tunnel; $S_{\max }$ is the maximum surface settlement; and $i$ is called the width of settlement trough (see Figure 1).

The Peck formula is needed to determine two parameters for the prediction of settlement: the ground loss $\left(V_{1}\right)$ and the width of settlement trough $(i)$. The ground loss $\left(V_{1}\right)$ is generally related to the construction process, the nature of the formation soil, and so on and can be determined based on empirical values or pretest sections. The width of settlement trough (i) determines the influence range of settlement and plays a major role in the amount of settlement when the ground loss is constant. The value of settlement trough width $(i)$ is related to the nature of the formation soil, the depth of the tunnel, and the radius of the tunnel. The literatures summarized the calculation formulas for various settlement trough width $(i)$, which is summarized in Table 1.

Numerous experimental and measured data show that there is no simple linear relationship between the width coefficient of the formation settlement trough and the tunnel depth of the stratum. The deformation of the overlying soil caused by tunnel excavation in different soil layers is different $[21,22]$. For clay layers with high cohesion, the disturbed soil is "pot" (see Figure 1) rather than inverted triangle shape. The complexity of the soil properties, the tunnel section size, the tunnel depth, and the construction method are different. Therefore, the expression of the width of settlement trough obtained by the researchers through the test or measured data is not uniform $[6,8,29-33]$. It is often only applied to a certain type or a certain area of the stratum, and there is little report on the theoretical solution of the width of the settlement trough.

Peck believed that ground volume $V_{1}$ is equal to the volume of the area bounded by the surface settlement curve and the original surface level, without considering the factors of seepage and formation consolidation, and can be obtained by integrating formula (1).

$$
V_{1}=S_{\max } \cdot \sqrt{2 \pi} i,
$$

where $V_{1}$ is numerically equal to the area of the pattern surrounded by the normal distribution curve $S_{(x)}$ and the horizontal line of the surface. 
TABLE 1: Calculation of width parameters of surface settlement trough.

\begin{tabular}{|c|c|c|c|}
\hline Document source & Applicable conditions & $\begin{array}{l}\text { Settlement trough width } \\
\text { (i) calculation formula }\end{array}$ & In accordance with \\
\hline Peck [6] & Various types of soil & $0.5(z / d)^{1-n} \times z,(n=0.8 \sim 1.0)$ & Measured statistics \\
\hline Attewell [7] & British clay & $0.5 z$ & Measured and model tested \\
\hline Han et al. [29] & $\begin{array}{l}\text { Cohesive soil, soft clay, and } \\
\text { hard clay in the London area }\end{array}$ & $(1-0.02 \phi) \times z$ & Measured statistics \\
\hline Atkinson and Potts [37] & $\begin{array}{l}\text { Loose sand } \\
\text { Compact superconsolidated clay }\end{array}$ & $\begin{array}{c}0.25(z+0.5 d) \\
0.25(1.5 z+0.25 d)\end{array}$ & Measured and model tested \\
\hline Lee et al. [38] & Clay & $0.29 z+0.5 d$ & Centrifuge test \\
\hline O’Reilly [39] & $\begin{array}{l}\text { Clay soil } \\
\text { Granular soil }\end{array}$ & $\begin{array}{l}i=0.43 z+1.1 \\
i=0.28 z-0.12\end{array}$ & Measured data \\
\hline Clough and Schmitdt [40] & British clay & $0.5(z / d)^{-0.2} \times z$ & Measured statistics \\
\hline
\end{tabular}

In the stochastic medium theory, the horizontal distance $r_{(z)}$ from the tunnel center to the surface settlement edge is called the surface settlement influence radius. Settlement occurs within the radius of impact of settlement, and settlement outside this range is negligible. By comparing the stochastic medium theory and Peck formula, Han et al. gave the relationship between the influence radius of settlement and the width of settlement trough as follows [29]:

$$
r_{(z)}=\sqrt{2 \pi} i .
$$

It can be seen from equations (2) and (3) that the area of the surface settlement trough is approximately equal to the area of the triangle with height $S_{\max }$ and base $2 \sqrt{2 \pi} i$. At the same time, it can be known from equation (3) that if the surface settlement influence radius $r_{(z)}$ can be obtained, the influence range of the settlement and the width of settlement trough of the tunnel excavation can be obtained.

\section{Calculation Method of the Influence Radius of Settlement Based on Slices Method}

There is a certain similarity between the mechanism of disturbed soil deformation and the instability mechanism of slope in urban subway tunnel construction. The excavation of the soil in the tunnel face breaks the original stress balance state of the stratum, and the soil within a certain range above the tunnel is deformed or even destabilized under the action of gravity. According to the theory of loosening earth pressure, there are potential sliding surfaces in tunnel excavation $[35,36]$. The formation process of this potential sliding surface is similar to that of slope stability analysis. Tunnel excavation is equivalent to unloading slope foot; because of the phenomenon of overexcavation, after the completion of tunnel support, there is still a gap between the supported soil and the supporting structure, and the soil will continue to deformation or move until it reaches an equilibrium state. The process of soil deformation or movement is simplified into the process of soil slices movement within the excavation disturbance range. Therefore, we can use the slope stability analysis method to analyze the surface deformation process of soil. The disturbance range of tunnel construction is limited, and the soil outside this range is not affected by construction disturbance. Therefore, as long as the position of the construction disturbance boundary surface can be determined, the horizontal distance from the tunnel center line to the disturbance boundary surface at different depths of the formation, that is, the influence radius of the settlement, can be calculated. Then, according to the relationship between the influence radius of settlement and the settlement trough width, the expression of the width of settlement trough can be obtained, and the settlement curves at different tunnel depths can be obtained.

The slices method is a commonly used method in the stability analysis of clay slopes. In view of the similarity between the mechanism of soil deformation caused by tunnel excavation and the mechanism of slope instability, this paper used slices method to determine the boundary of tunnel construction disturbance. In order to simplify the analysis process, the following two assumptions are made: (1) The soil is homogeneous, and the cohesion and internal friction angles of the various soil strips formed by the strips are the same. The vertical line at the center of the tunnel is the axis of symmetry for the deformation of soil mass. (2) As long as a point in the soil mass slides, it is considered that the soil at that point has reached the failure, that is, the soil at the disturbance boundary is considered to be in a limit equilibrium state. Due to the symmetry of soil disturbed by tunnel construction, in order to simplify the calculation process, half of the soil is taken for analysis, as shown in Figure 2.

Assume that the disturbance boundary is a circular arc with the point $O$ as the center and $R$ as the radius ( $a b$ in Figure 2), and the point $O$ of the arc center is directly above the centerline of the tunnel. The soil within the disturbance range is divided into $n$ soil strips of equal width. If any of the $i$ th soil strips (shaded soil strips in Figure 2) is used as the isolation object for force analysis, the force acting on the soil strips has the self-weight $W_{i}$ and the road load $Q_{i}$. The normal reaction force $N_{i}$ and the tangential reaction force $T_{i}$ on the bottom surface $d e$, the normal forces $E_{1 i}$ and $E_{2 i}$, and the tangential forces $F_{1 i}$ and $F_{2 i}$ are shown in Figure 3. To simplify the calculation, it is assumed that the resultant force of $E_{1 i}$ and $\mathrm{F}_{1 i}$ is opposite to the combined force of $E_{2 i}$ and $F_{2 i}$ and acts on the same straight line, and the two cancel each other out.

According to the static balance condition of the soil strip, there is 


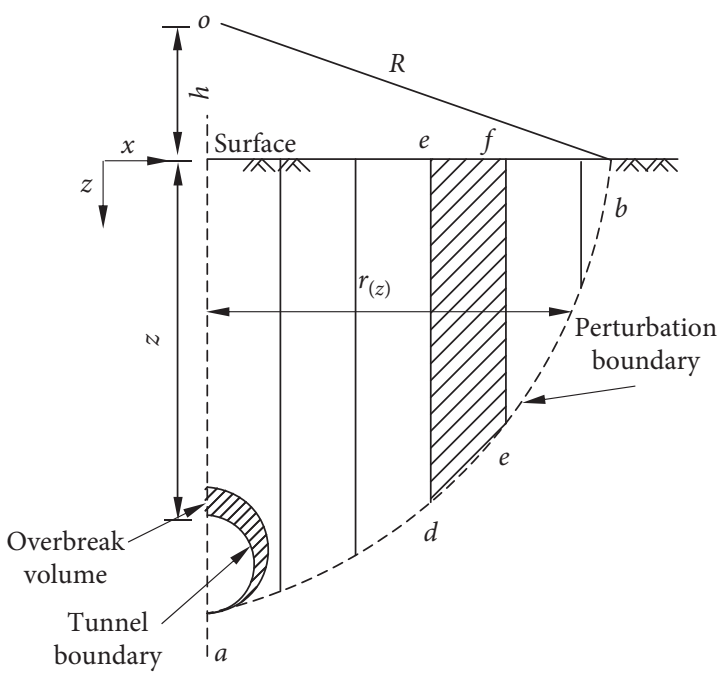

Figure 2: The analysis based on the slices method.

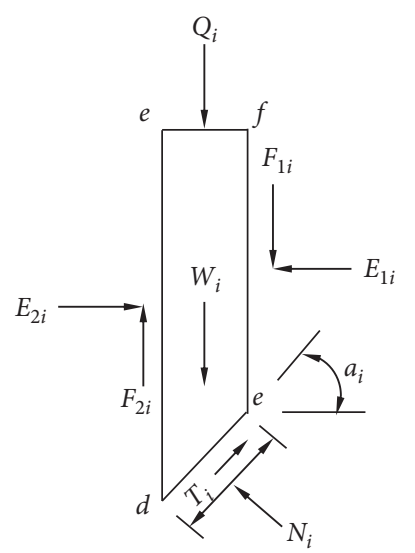

FIgURE 3: Stress analysis of a soil slice.

$$
\begin{aligned}
N_{i} & =\left(W_{i}+Q_{i}\right) \cos \alpha_{i}, \\
T_{i} & =\left(W_{i}+Q_{i}\right) \sin \alpha_{i},
\end{aligned}
$$

where $\alpha_{i}$ is the angle between $T_{i}$ and the horizontal plane.

Let the length of the arc $d e$ be $l_{i}$, then the normal stress and shear stress acting on de are, respectively,

$$
\begin{gathered}
\sigma_{i}=\frac{N_{i}}{l_{i}}=\frac{\left(W_{i}+Q_{i}\right) \cos \alpha_{i}}{l_{i}}, \\
\tau_{i}=\frac{T_{i}}{l_{i}}=\frac{\left(W_{i}+Q_{i}\right) \sin \alpha_{i}}{l_{i}} .
\end{gathered}
$$

In addition, according to the Coulomb theory, the shear force generated on the arc de is

$$
S_{i}=\left(c_{i}+\sigma_{i} \tan \varphi_{i}\right) \cdot l_{i},
$$

where $c_{i}$ is the cohesion of the layer at the bottom of the soil strip and $\varphi_{i}$ is the internal friction angle of the local layer.

Since the tunnel construction will excavate the bottom of the soil strip within the excavation range, the shearing force and shear resistance of the bottom surface of the strip will not exist. Then, the total shear force acting on the entire disturbance boundary $a b$ is

$$
T=\sum_{i=m}^{n} T_{i}=\sum_{i=m}^{n}\left(W_{i}+Q_{i}\right) \sin \alpha_{i}
$$

where $m=r / d$ is the tunnel radius and $d$ is the width of the bar.

With the continuous refinement of the scores, the aforementioned formula becomes the integral in the general sense.

$$
\begin{aligned}
T & =\int_{r}^{\sqrt{R^{2}-h^{2}}} \gamma z \frac{x}{R} \mathrm{~d} x \\
& =\int_{\theta_{1}}^{\theta_{0}} \gamma R \sin \theta \cos \theta \mathrm{d}(R \cos \theta)-\int_{r}^{\sqrt{R^{2}-h^{2}}} \gamma h \frac{x}{R} \mathrm{~d} x, \\
& =\int_{\theta_{0}}^{\theta_{1}} \gamma R^{2} \sin ^{2} \theta \cos \theta \mathrm{d} \theta-\frac{\gamma h\left(R^{2}-h^{2}-r^{2}\right)}{2 R}, \\
& =\frac{\gamma\left(R^{2}-r^{2}\right)^{3 / 2}}{3 R}-\frac{\gamma h^{3}}{3 R}-\frac{\gamma h\left(R^{2}-h^{2}-r^{2}\right)}{2 R}, \\
& =\frac{\gamma}{6 R}\left[2\left(R^{2}-r^{2}\right)^{3 / 2}-\left(3 R^{2}-h^{2}-3 r^{2}\right) h\right]
\end{aligned}
$$

where $\sin \theta_{0}=h / R, \cos \theta_{1}=r / R$.

The total shear force produced over the entire disturbance boundary $a b$ is

$$
\begin{aligned}
& S=\sum_{i=m}^{n} S_{i}=\sum_{i=m}^{n}\left(c_{i}+\sigma_{i} \tan \phi_{i}\right) \cdot l_{i}, \\
& S=c \cdot\left(\theta_{1}-\theta_{0}\right) R+\int_{r}^{\sqrt{R^{2}-h^{2}}} \gamma z \frac{z+h}{R} \tan \varphi \mathrm{d} x,
\end{aligned}
$$$$
=\left(\theta_{1}-\theta_{0}\right) R c+\int_{\theta_{1}}^{\theta_{0}} \gamma(R \sin \theta-h) \sin \theta \tan \varphi \mathrm{d}(R \cos \theta),
$$$$
=\left(\theta_{1}-\theta_{0}\right) R c+\int_{\theta_{0}}^{\theta_{1}} \gamma R(R \sin \theta-h) \sin ^{2} \theta \tan \varphi \mathrm{d} \theta,
$$$$
=\left(\theta_{1}-\theta_{0}\right) R c+\gamma R \tan \varphi\left[R\left(\frac{\cos ^{3} \theta_{1}-\cos ^{3} \theta_{0}}{3}+\cos \theta_{0}-\cos \theta_{1}\right)\right.
$$$$
\left.-h\left(\frac{\theta_{1}-\theta_{0}}{2}-\frac{\sin 2 \theta_{1}-\sin 2 \theta_{0}}{4}\right)\right] \text {, }
$$$$
=\left(\theta_{1}-\theta_{0}\right) R c+\gamma R \tan \varphi\left[\frac{r^{3}}{3 R^{2}}-\frac{R}{3}\left(1-\frac{h^{2}}{R^{2}}\right)^{3 / 2}+\sqrt{R^{2}-h^{2}}-r\right.
$$$$
\left.+\frac{h}{2}\left(\frac{r}{R} \sqrt{1-\frac{r^{2}}{R^{2}}}-\frac{h}{R} \sqrt{1-\frac{h^{2}}{R^{2}}}-\theta_{1}+\theta_{0}\right)\right] \text {. }
$$

The ratio of $S$ to $T$ can be considered as the safety factor $F_{\mathrm{s}}$, that is, $F_{\mathrm{s}}=S / T$. When the total shear force Tacting on the entire disturbance boundary $a b$ is equal to the total shear force $S$, that is, $F_{\mathrm{s}}=1$. The soil within the boundary is 
deformed by the tunnel construction disturbance, while soil mass outside the boundary is not affected by construction disturbance and remains static.

Therefore, the position of the construction disturbance boundary can be determined according to equations (6) and (8). Taking the intersection of the centerline of the tunnel and the ground as the coordinate origin and establishing the coordinate system with the horizontal direction as the $x$ direction and the vertical direction as the $z$ direction, the expression of the disturbance boundary can be expressed as

$$
x^{2}+(z+h)^{2}=R^{2} \text {, }
$$

where $h$ is the distance from the center of the arc to the ground.

According to formula (9), it is possible to determine the influence radius $r_{(z)}$ of the settlement at different depths:

$$
r_{(z)}=x=\sqrt{R^{2}-(z+h)^{2}} \text {. }
$$

Then, according to formula (3), the width of settlement trough at different buried depths is determined, and then the settlement curves of the corresponding formation are obtained by formulas (1) and (2).

\section{Case Study}

In order to prove the rationality of the method, the theoretical calculation results and the measured data were compared with the Heathrow Express tunnel (sand layer) and the Green Park tunnel (clay formation). Stratum parameters of the two tunnels are shown in Table 2.

Figure 4(a) shows the comparison between the surface settlement curve calculated by the method and the measured settlement data in the sand layer. The surface settlement curve calculated in this paper is in good agreement with the measured surface settlement data, and the error is small. It is indicated that in the sand layer, the calculation method of surface settlement based on the slices method proposed in this paper has certain rationality. Figure 4(b) shows the comparison of the settlement values of the formations at different depths above the tunnel centerline calculated by the method and the measured data. Meanwhile, the settlement values of different tunnel depths above the tunnel center line are calculated by using the methods proposed by Mair et al. [8] and Han et al. [29]. The settlement calculation formula proposed in this paper can accurately reflect the variation trend of the settlement with the tunnel depth above the tunnel centerline, and the calculation results are in good agreement with the measured data. The method proposed by Mair and Han is more accurate for the prediction of surface settlement. However, as the depth of the stratum increases, the difference between the calculated results and the measured data is larger and larger, and the calculation results are significantly larger than the measured results. It is indicated that in the sand layer, the settlement calculation formula proposed in this paper more accurately reflects the variation trend of the settlement with the tunnel depth above the centerline of the tunnel compared with the other two methods.
Figure 5(a) shows the comparison between the land settlement curve calculated by the method and the measured data. As can be seen from the figure, the calculated results are in good agreement with the measured results. It is indicated that in the clay stratum, the calculation method of settlement based on the slices method proposed in this paper has certain rationality. Figure 5(b) shows the comparison between the settlement and the measured results at different depths above the tunnel centerline calculated by the method proposed by Mair et al. [8] and the method proposed by Han et al. [29]. In the clay stratum, this paper proposed that the settlement calculation formula can accurately predict the formation settlement outside the depth of $4 d$ and predicted that there is a large error in the surface settlement within the $4 d$ range, while the error of Mair and Han method is relatively small. It is indicated that in the clay stratum, the settlement calculation formula can predict the surface settlement and shallow stratum settlement more accurately and there is a big error in predicting the stratum settlement within $4 d$ near the tunnel vault.

\section{Analysis and Discussion}

In order to further prove the rationality and feasibility of the formula proposed in this paper, this paper analyzed the parameters of sand and clay formations often encountered in urban subway construction.

The diameter of the tunnel is $d=6,8,10$, and $12 \mathrm{~m}$, and the ratio of tunnel depth to diameter is $z / d=1,1.5,2.0,2.5$, and 3.0. In the sand layer, the cohesion $c=0$, the internal friction angle $\varphi=20^{\circ}, 30^{\circ}, 40^{\circ}$, and $50^{\circ}$. The relationship between the influence radius of the settlement $\left(r_{(z)}\right)$ and the ratio of the tunnel depth to the diameter is shown in Figure 6(a); in clay formation, internal friction angle $\varphi=10^{\circ}$ and cohesion $c=20 \mathrm{kPa}, 30 \mathrm{kPa}, 40 \mathrm{kPa}$, and $50 \mathrm{kPa}$. Figure 6(b) shows the relationship between $r_{(z)}$ and $(z / d)$.

As shown in Figure 6(a), when the diameter of the tunnel is less than or equal to $8 \mathrm{~m}$, the influence radius increases as $z / d$ increases. When the diameter of the tunnel is greater than or equal to $10 \mathrm{~m}$, when $z / d<1.5$, the radius of influence increases with the increase of $z / d$; when $1.5<z / d<2.0$, the influence radius decreases with the increase of $z / d$; and when $2.0<z / d$, the radius of influence is zero. It is indicated that as the tunnel depth and internal friction angle increase, the disturbance caused by tunnel excavation will not develop to the ground surface. As shown in Figure 6(b), when the diameter of the tunnel is less than or equal to $6 \mathrm{~m}$, the influence radius increases as $z / d$ increases. When the diameter of the tunnel is greater than $6 \mathrm{~m}$, the radius of influence increases first with $z / d$ and then decreases to zero. It is indicated that as the tunnel depth and cohesion increase, the disturbance caused by tunnel excavation will not develop to the surface. The calculated influence radius is 0 , which does not mean that there is no surface settlement, which means that the calculation formula in this paper is not applicable to this case. The sliding surface caused by tunnel excavation and developed to the surface is the precondition for deriving the formula. When the tunnel depth increases and the formation conditions are good, the tunnel 
TABLE 2: Formation parameters of the Heathrow Express and Green Park tunnels.

\begin{tabular}{lccccccc}
\hline Name & $r(\mathrm{~m})$ & $z_{0}(\mathrm{~m})$ & $\gamma\left(\mathrm{kN} / \mathrm{m}^{3}\right)$ & $c(\mathrm{kPa})$ & $\phi\left({ }^{\circ}\right)$ & $R(\mathrm{~m})$ & $r_{(z)}(\mathrm{m})$ \\
\hline Heathrow Express tunnel [11] & 4.25 & 19.0 & 19 & 12 & 28 & 26.8 & 26.2 \\
Green Park tunnel [11] & 2.07 & 29.4 & 19 & 175 & - & 34.1 & 34 \\
\hline
\end{tabular}

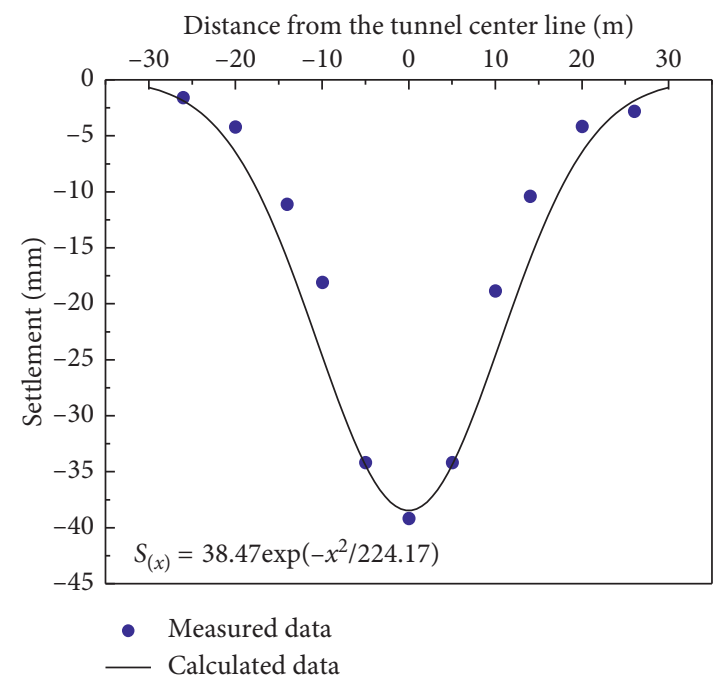

(a)

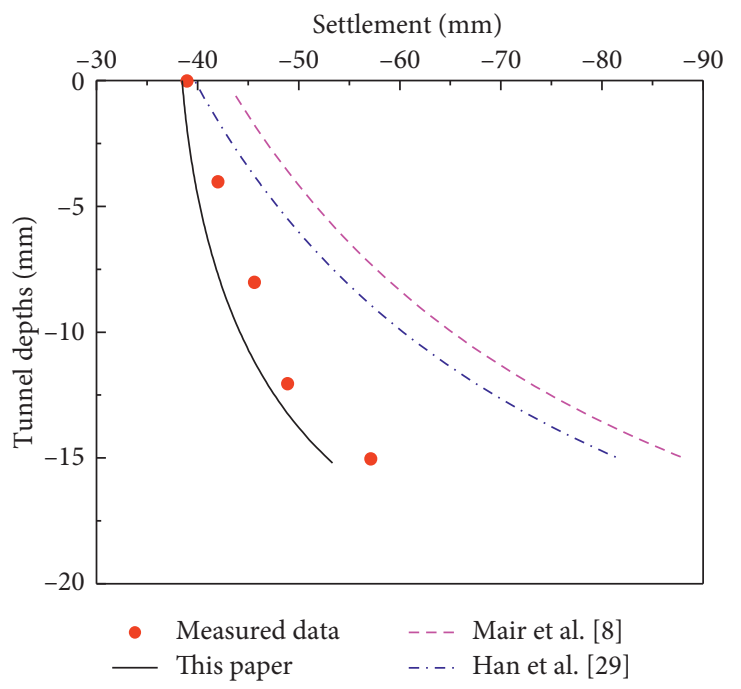

(b)

Figure 4: (a) Surface settlement curve and (b) stratum settlement above the center line, the Heathrow Express tunnel.

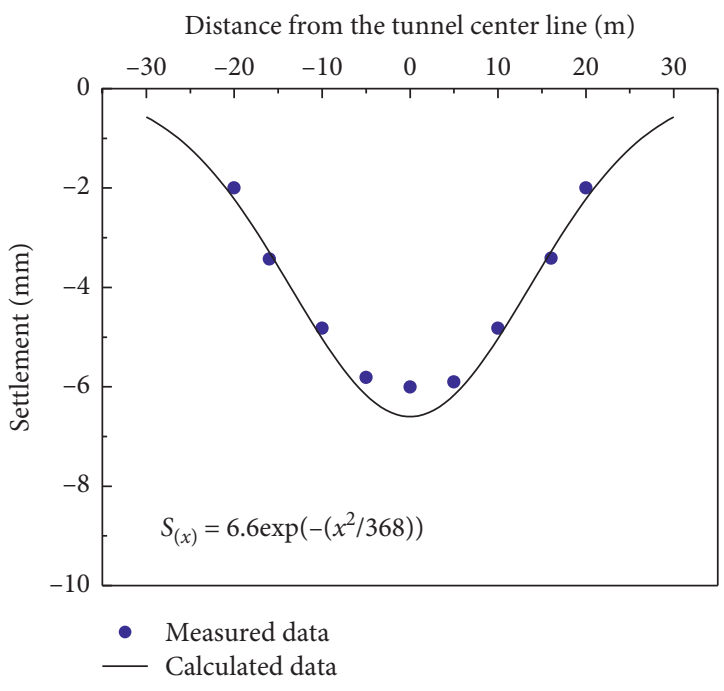

(a)

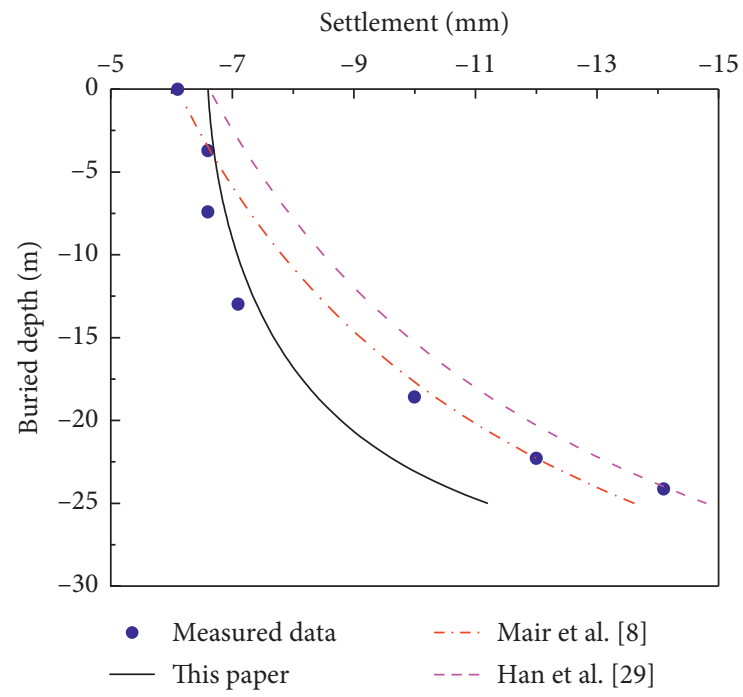

(b)

Figure 5: (a) Surface settlement curve and (b) stratum settlement above the center line, the Green Park tunnel.

excavation disturbance will not affect the surface and forms a sliding surface, so the formula in this paper is not applicable.

In the construction process of the tunnel, in order to reduce the surface settlement, the excavation face is usually reinforced. Reinforcement of the excavation face is equivalent to applying a force to prevent the sliding of the soil strip where the tunnel is located and improving the stability of the slope. However, whether the reinforcement measures for the excavation face can reduce the radius of influence requires further analysis. The safety factor is used to characterize the size of the reinforcement, and the relationship between the safety factor and the influence radius is analyzed. When the safety factor of the slope takes different values, the relationship between the influence radius and the internal friction angle and cohesion is shown in Figure 7.

As shown in Figure 7(a), in the sand layer, the influence radius decreases as the internal friction angle increases and decreases as the safety factor increases. As shown in 


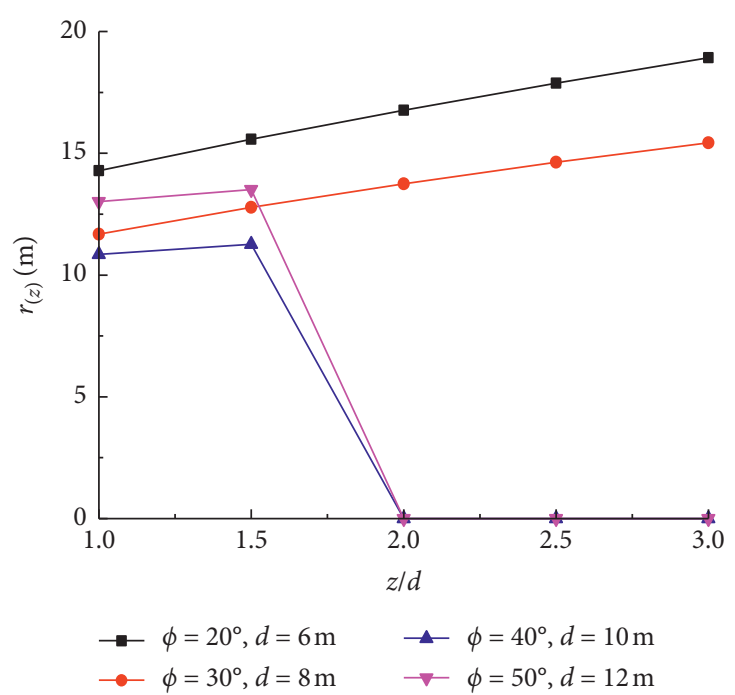

(a)

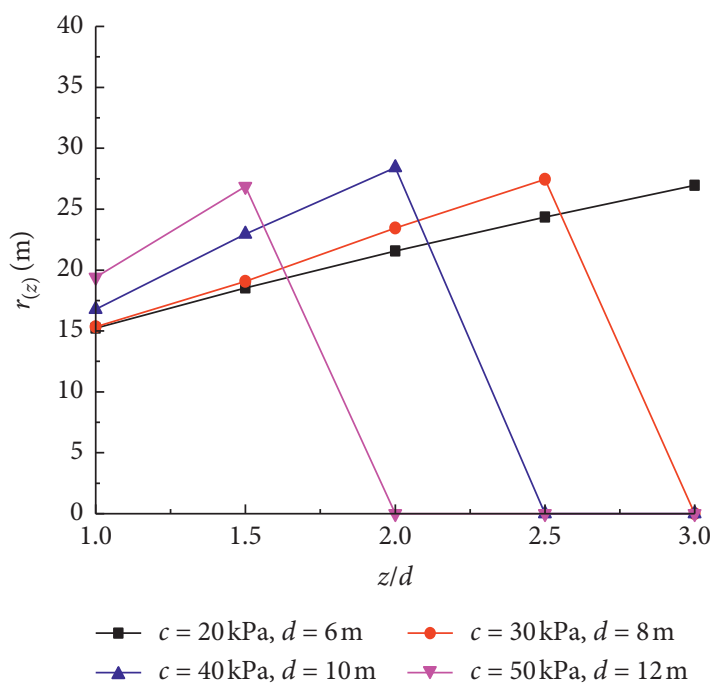

(b)

Figure 6: Trend of $r_{(z)}$ with $z / d$. (a) Sand. (b) Clay.

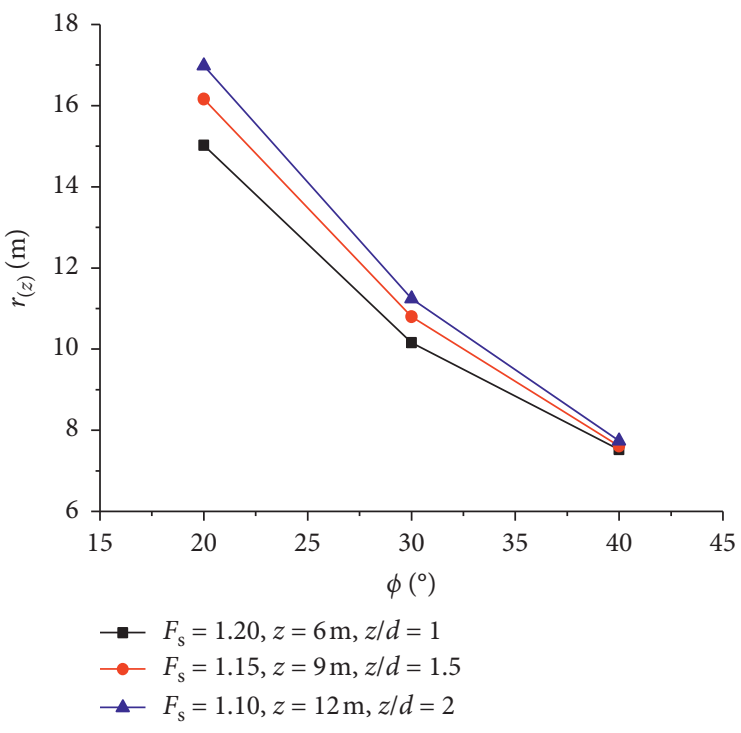

(a)

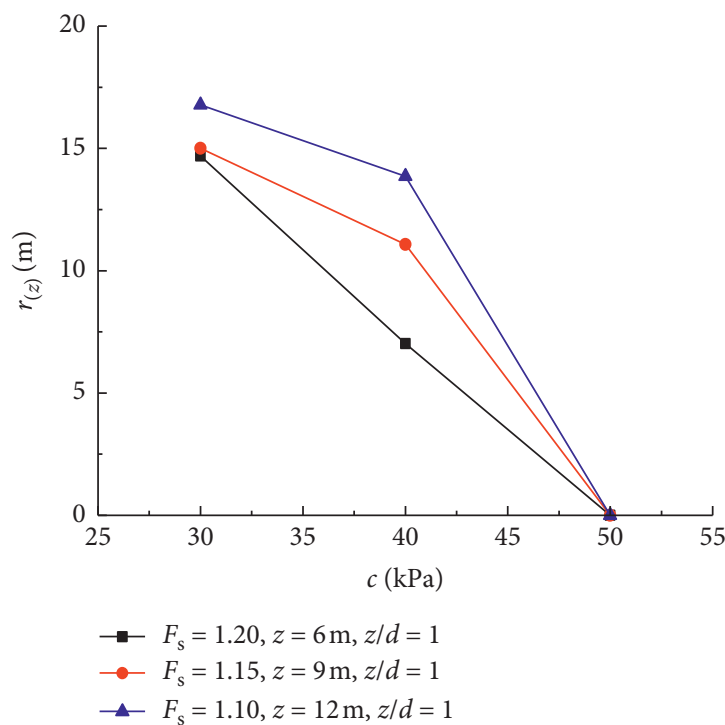

(b)

Figure 7: Trend of $r_{(z)}$ with $\phi$ and $c$. (a) Sand. (b) Clay.

Figure $7(b)$, in the clay formation, the influence radius decreases as the cohesion increases and decreases as the safety factor increases. It is indicated that in sand, when the internal friction angle is less than $40^{\circ}$, the reinforcement of the excavation face can reduce the radius of influence, and the radius of influence can also be reduced by increasing the internal friction angle of the formation. In clay formations, when the cohesion is less than $50 \mathrm{kPa}$, the reinforcement of the excavation face can reduce the radius of influence, and the radius can also be reduced by increasing the cohesive of the formation. It can also be seen from Figure 7(a) that as the internal friction angle increases, the amplitude of the radius of influence decreases. In the sand layer, when the internal friction angle is greater than $40^{\circ}$, reinforcement measures are applied to the excavation face to reduce the influence radius. In the clay formation, when the cohesion is greater than $50 \mathrm{kPa}$, reinforcement measures are applied to the excavation face, and the radius of influence cannot be reduced.

\section{Conclusions}

Based on the slices method, the calculation formula for predicting the influence radius of surface settlement was established. The rationality of the formula was verified by engineering examples and discussion. The conclusions obtained are as follows: 
(1) Based on the slices method, a formula for predicting the influence radius of the surface settlement and the width of the settlement trough was established.

(2) In the sand layer, the settlement calculation formula proposed in this paper more accurately reflects the variation trend of the settlement with the tunnel depth above the centerline of the tunnel compared with the other two methods. In the clay stratum, this paper proposed that the settlement calculation formula can predict ground settlement and shallow stratum settlement more accurately, and there is a big error in predicting the stratum settlement within $4 d$ near the tunnel vault.

(3) In the sand layer, the internal friction angle is less than $40^{\circ}$, and the reinforcement applied to the excavation face can reduce the radius of influence; when the internal friction angle is greater than $40^{\circ}$, reinforcement measures for the excavation face cannot reduce the radius of influence. In the clay formation, when the cohesion is less than $50 \mathrm{kPa}$, the reinforcement for the excavation face can reduce the radius of influence; when the cohesion is greater than $50 \mathrm{kPa}$, the reinforcement surface is applied to the excavation face, and the radius of influence cannot be reduced.

\section{Data Availability}

The data used to support the findings of this study are included within the article.

\section{Conflicts of Interest}

The authors declare that they have no conflicts of interest.

\section{Acknowledgments}

The present work was subsidized and supported by the National Natural Science Foundation of China (no. 51578447), the Science and Technology Project of Ministry of Housing Urban-Rural Construction (no. 2017-K4-032), the China Postdoctoral Science Foundation (no. 2018M643809XB), and the Natural Science Basic Research Program of Shaanxi (no. 2019JQ-762). The financial supports are gratefully acknowledged by the authors.

\section{References}

[1] Z. Zhang, M. Huang, C. Zhang, K. Jiang, and M. Lu, "Timedomain analyses for pile deformation induced by adjacent excavation considering influences of viscoelastic mechanism," Tunnelling and Underground Space Technology, vol. 85, no. 3, pp. 392-405, 2019.

[2] Z. P. Song, S. H. Li, X. G. Zhang, J. B. Wang, and T. Wang, "Study on strata settlement regular pattern induced by tunnel construction based on Peck formula," Xi'an University of Architecture and Technology, vol. 50, no. 2, pp. 190-195, 2018.

[3] X. X. Tian, Z. P. Song, and J. B. Wang, "Study on the propagation law of tunnel blasting vibration in stratum and blasting vibration reduction technology," Soil Dynamics and Earthquake Engineering, vol. 126, Article ID 105813, 2019.

[4] Y. W. Zhang, X. L. Weng, Z. P. Song, and Y. F. Sun, "Modeling of loess soaking induced impacts on metro tunnel using water soaking system in centrifuge," Geofluids, vol. 2019, Article ID 5487952, 17 pages, 2019.

[5] Y. Cheng, Z. Song, J. Jin, and T. Yang, "Attenuation characteristics of stress wave peak in sandstone subjected to different axial stresses," Advances in Materials Science and Engineering, vol. 2019, Article ID 6320601, 11 pages, 2019.

[6] R. B. Peck, "Deep excavations and tunnelling in soft ground," in Proceedings of the 7th International Conference on Soil Mechanics and Foundation Engineering, pp. 225-290, Mexico City, Mexico, 1969.

[7] P. B. Attewell and J. P. Woodman, "Predicting the dynamics of ground settlement and its derivatives caused by tunneling in soil," Ground Engineering, vol. 15, no. 8, pp. 9-36, 1982.

[8] R. J. Mair, R. N. Taylor, and A. Bracegirdle, "Subsurface settlement profiles above tunnels in clays," Géotechnique, vol. 45, no. 2, pp. 361-362, 1995.

[9] C. Sagaseta, "Analysis of undraind soil deformation due to ground loss," Géotechnique, vol. 37, no. 3, pp. 301-320, 1987.

[10] A. Verruijt and J. R. Booker, "Surface settlements due to deformation of a tunnel in an elastic half plane," Géotechnique, vol. 46, no. 4, pp. 753-756, 1996.

[11] N. Loganathan and H. G. Poulos, "Analytical prediction for tunneling-induced ground movements in clays," Journal of Geotechnical and Geoenvironmental Engineering, vol. 124, no. 9, pp. 846-856, 1998.

[12] Z.-P. Song, S.-H. Li, J.-B. Wang, Z.-Y. Sun, J. Liu, and Y.-Z. Chang, "Determination of equivalent blasting load considering millisecond delay effect," Geomechanics and Engineering, vol. 15, no. 2, pp. 745-754, 2018.

[13] R. D. Mindlin and D. H. Cheng, "Nuclei of strain in the semiinfinite solid," Journal of Applied Physics, vol. 21, no. 9, pp. 926-930, 1950.

[14] A. M. M. Wood, "The circular tunnel in elastic ground," Géotechnique, vol. 25, no. 1, pp. 115-127, 1975.

[15] A. Bobet, "Analytical solutions for shallow tunnels in saturated ground," Journal of Engineering Mechanics, vol. 127, no. 12 , pp. 1258-1266, 2001.

[16] K. H. Park, "Elastic solution for tunneling-induced ground movements in clays," International Journal of Geomechanics, vol. 4, no. 4, pp. 310-318, 2004.

[17] K.-H. Park, "Analytical solution for tunnelling-induced ground movement in clays," Tunnelling and Underground Space Technology, vol. 20, no. 3, pp. 249-261, 2005.

[18] A. M. Puzrin, J. B. Burland, and J. R. Standing, "Simple approach to predicting ground displacements caused by tunnelling in undrained anisotropic elastic soil," Géotechnique, vol. 62, no. 4, pp. 341-352, 2012.

[19] J. Litwiniszyn, "The theories and model research of movements of ground masses," in Proceedings of the European Congress Ground Movement, pp. 203-209, Leeds, UK, April 1957.

[20] J. S. Yang, B. C. Liu, and M. C. Wang, "Modeling of tunnelinginduced ground surface movements using stochastic medium theory," Tunnelling and Underground Space Technology, vol. 19, no. 2, pp. 113-123, 2004.

[21] X. L. Yang and J. M. Wang, "Ground movement prediction for tunnels using simplified procedure," Tunnelling and Underground Space Technology, vol. 26, no. 3, pp. 462-471, 2011.

[22] R. J. Finno, I. S. Harahap, and P. J. Sabatini, "Analysis of braced excavations with coupled finite element formulations," Computers and Geotechnics, vol. 12, no. 2, pp. 91-114, 1991. 
[23] T. Wang, Z. P. Song, J. Y. Yang, J. Wang, and X. Zhang, "Experimental research on dynamic response of red sandstone soil under impact loads," Geomechanics and Engineering, vol. 17, no. 4, pp. 393-403, 2019.

[24] D. J. White, W. A. Take, and M. D. Bolton, "Soil deformation measurement using particle image velocimetry (PIV) and photogrammetry," Géotechnique, vol. 53, no. 7, pp. 619-631, 2003.

[25] G. Song and J. A. Black, "Soil displacement due to tunnelling using small-scale centrifuge technology," in Proceedings of the 3rd European Conference on Physical Modelling in Geotechnics, ECPMG 2016, Nantes, France, June 2016.

[26] Z. P. Song, J. C. Mao, X. X. Tian, Y. W. Zhang, and J. B. Wang, "Optimization analysis of controlled blasting for passing through houses at close range in super-large section tunnels," Shock and Vibration, vol. 2019, Article ID 1941436, 16 pages, 2019, In press.

[27] T. E. Vorster, A. Klar, K. Soga, and R. J. Mair, "Estimating the effects of tunneling on existing pipelines," Journal of Geotechnical and Geoenvironmental Engineering, vol. 131, no. 11, pp. 1399-1410, 2005.

[28] T. B. Celestino, R. A. M. P. Gomes, and A. A. Bortolucci, "Errors in ground distortions due to settlement trough adjustment," Tunnelling and Underground Space Technology, vol. 15, no. 1, pp. 97-100, 2000.

[29] X. Han, N. Li, and J. R. Standing, "An adaptability study of Gaussian equation applied to predicting of ground settlements induced by tunneling in China," Rock and Soil Mechanics, vol. 28, no. 1, pp. 23-28, 2007, in Chinese.

[30] X. L. Yang and F. Huang, "Three-dimensional failure mechanism of a rectangular cavity in a Hoek-Brown rock medium," International Journal of Rock Mechanics and Mining Sciences, vol. 61, no. 10, pp. 189-195, 2013.

[31] M. Fraldi and F. Guarracino, "Evaluation of impending collapse in circular tunnels by analytical and numerical approaches," Tunnelling and Underground Space Technology, vol. 26, no. 4, pp. 507-516, 2011.

[32] G. Mollon, D. Dias, and A.-H. Soubra, "Rotational failure mechanisms for the face stability analysis of tunnels driven by a pressurized shield," International Journal for Numerical and Analytical Methods in Geomechanics, vol. 35, no. 12, pp. 1363-1388, 2011.

[33] R. J. Mair, "Bored tunnelling in the urban environment," in Proceedings of the 14th International Conference on Soil Mechanics and Foundation Engineering, Hamburg, Germany, September 1997.

[34] A. R. Selby, "Surface movements caused by tunnelling in twolayer soil," Geological Society, London, Engineering Geology Special Publications, vol. 5, no. 1, pp. 71-77, 1988.

[35] K. Terzaghi, Theoretical Soil Mechanics, John Wiley \& Sons, New York, NY, USA, 1943.

[36] Z. W. Fan and Z. X. Zhang, "Model test of excavation face stability of EPB shield in sandy cobble ground and adjacent building effect," Chinese Journal of Rock Mechanics and Engineering, vol. 32, no. 12, pp. 2506-2512, 2013, in Chinese.

[37] J. H. Atkinson and D. M. Potts, "Settlement above shallow tunnels in soft ground," ASCE Journal of the Geotechnical Engineering Division, vol. 103, no. 4, pp. 307-325, 1977.

[38] C. J. Lee, B. R. Wu, and S. Y. Chiou, "Soil movements around a tunnel in soft soils," Proceedings of the National Science Council, Part A, Physical Science and Engineering, vol. 23, no. 2, pp. 235-247, 1999.

[39] M. P. O' Reilly and B. M. New, "Settlement above tunnels in the United Kingdom-their magnitude and predication," in
Tunnelling 82, M. Jones, Ed., pp. 173-181, Institution of Mining and Metallurgy, London, UK, 1982.

[40] G. W. Clough and B. Schmitdt, "Design and performance of excavation and tunnels in soft clay," in Soft Clay Engineering, pp. 569-634, Elsevier, Amsterdam, Netherlands, 1981. 